\title{
Contribución para el perfeccionamiento del diseño, la construcción y la operación de reactores UASB aplicados al tratamiento de aguas residuales urbanas - Parte 2: Tratamiento preliminar, bombeo y distribución de caudal
}

\section{Contribution for improving the design, construction and operation of UASB reactors treating sewage - Part 2: Preliminary treatment, pumping and influent distribution}

- Fecha de entrada: 01/08/2018

- Fecha de aprobación: $17 / 09 / 2018$

Thiago Bressani Ribeiro / Lívia Cristina da Silva Lobato / Saulo Nonato de Souza / Eduardo Sabino Pegorini / Carlos Augusto de Lemos Chernicharo (*)

DOI: $10.4322 /$ dae.2018.039es

\section{Resumen}

La etapa de tratamiento preliminar es fundamental para el adecuado acondicionamiento del agua residual antes de su ingreso a los reactores UASB. El objetivo de esta Nota Técnica es discutir acerca de los principales problemas, sus respectivos orígenes y las posibles soluciones asociadas al tratamiento preliminar, bombeo y distribución de caudal en los reactores UASB. En síntesis, un adecuado tratamiento preliminar influenciará positivamente en: i) el mantenimiento de las estructuras de distribución de caudal y canaletas colectoras de efluente, evitando obstrucciones; ii) en la reducción de sedimentación y acumulación de arena en el fondo de los reactores UASB; iii) en el manejo del lodo, de modo a obtener un material sólido con menor contenido de residuos y arena y de mejor calidad estética; iv) en el manejo de la nata, sobre todo debido a que, cuando algunos materiales no son removidos adecuadamente en el tratamiento preliminar, tienden a flotar en el interior del reactor y a acumularse en la parte superior del separador trifásico (por ejemplo: plásticos o residuos livianos en general); y v) en la reducción significativa de problemas comunes asociados a la operación de unidades de procesamiento mecanizado de lodo y nata, tal como la paralización por obstrucciones de tuberías o la falla y pérdida de rendimiento de equipos debido al desgaste. Palabras clave: Cribado, desarenador, olor, residuos de tratamiento preliminar, tamizaje

\footnotetext{
Thiago Bressani Ribeiro - Ingeniero Ambiental de la Universidad Fundación Minera de Educación y Cultura (FUMEC), Brasil. Máster en Saneamiento, Medio Ambiente y Recursos Hídricos de la UFMG, Brasil. Doctorando en Saneamiento de la Universidad de Ghent, Bélgica y de la UFMG, Brasil. Miembro del Instituto Nacional de Ciencia y Tecnología en Plantas Sostenibles de Tratamiento de Aguas Residuales Urbanas (INCT ETEs Sustentáveis por sus siglas en portugués).

Lívia Cristina da Silva Lobato - Ingeniera Civil de la Universidad Federal de Minas Gerais (UFMG), Brasil. Doctora en Saneamiento, Medio Ambiente y Recursos Hídricos de la UFMG, Brasil. Miembro del Instituto Nacional de Ciencia y Tecnología en Plantas Sostenibles de Tratamiento de Aguas Residuales Urbanas (INCT ETEs Sustentáveis por sus siglas en portugués).

Saulo Nonato de Souza - Ingeniero Civil de la Universidad Federal de Minas Gerais (UFMG), Brasil. Ingeniero de Operación de la Compañía de Saneamiento de Minas Gerais (COPASA), Brasil. Estudiante de Maestría en Saneamiento, Medio Ambiente y Recursos Hídricos de la UFMG, Brasil

Eduardo Sabino Pegorini - Ingeniero Agrónomo de la Universidad del Paraná (UFPR), Brasil. Máster en Ciencias del Suelo de la UFPR, Brasil. Ingeniero de la Unidad de Proyecto KFW en la Compañía de Saneamiento de Paraná (Sanepar), Brasil.

Carlos Augusto de Lemos Chernicharo - Ingeniero Civil de la Universidad Federal de Minas Gerais (UFMG), Brasil. Doctor en Ingeniería Ambiental de la Universidad de Newcastle upon Tyne, UK. Profesor titular del Departamento de Ingeniería Sanitaria y Ambiental de la UFMG, Brasil. Coordinador del Instituto Nacional de Ciencia y Tecnología en Plantas Sostenibles de Tratamiento de Aguas Residuales Urbanas (INCT ETEs Sustentáveis por sus siglas en portugués). (*) Dirección para correspondencia: Avenida Antônio Carlos 6.627 - Universidade Federal de Minas Gerais - Escola de Engenharia - Departamento de Engenharia Sanitária e Ambiental - Bloco 1. Belo Horizonte, MG. CEP: 31270-901. e-mail: calemosadesa.ufmg.br
} 


\section{Abstract}

The preliminary treatment step is essential for the adequate sewage conditioning prior to UASB reactors. This Technical Note aims to discuss the main problems, their respective origins and the possible improvements associated with the preliminary treatment, pumping and influent distribution to UASB reactors. In summary, an adequate preliminary treatment will positively influence: i) the maintenance of the flow distribution structures and effluent collection system, avoiding obstructions; ii) the reduction of deposition and accumulation of sand at the bottom of UASB reactors; iii) the management of sludge, since it will be possible to obtain a biosolid with lower content of debris and sand, and of better aesthetic quality; iv) the management of scum, mainly due to the fact that, when not properly removed in the preliminary treatment, some materials (e.g. plastics and light debris in general) tend to float inside the reactor and accumulate in the upper part of the three-phase separator; v) the significant reduction of common problems in the operation of sludge and scum processing units, such as stoppage due to pipe obstruction, breakage and loss of equipment performance due to wear. Keywords: Grit chamber, odour, residues of the preliminary treatment, screening, sieving

\section{INTRODUCCIÓN}

En una planta de tratamiento de aguas residuales urbanas (PTAR), la etapa de tratamiento preliminar (TP) está compuesta típicamente por rejillas de barras y desarenadores. El principal papel de las unidades que componen el tratamiento preliminar es la remoción de sólidos que podrían comprometer el adecuado funcionamiento de las unidades posteriores del tratamiento - en este caso, los reactores anaerobios de manto de lodo y flujo ascendente (UASB, por sus siglas del inglés Upflow Anaerobic Sludge Blanket), sobre todo debido a las obstrucciones de tuberías, averías en equipamientos electromecánicos, acumulación de arena en el interior de los reactores y formación de nata. Esos problemas usualmente también se extienden a equipos y unidades de procesamiento de subproductos del tratamiento, como el lodo y la nata, generando paralizaciones frecuentes; las cuales pueden variar desde una simple limpieza y desobstrucción hasta la falla y daño de equipos de mantenimiento delicado y lento (por ejemplo: centrífugas).

Con respecto al cribado (separación con rejillas), el objetivo es adoptar un espaciamiento entre las barras que permita una remoción eficiente de residuos sólidos (por ejemplo: cabello, hisopos, toallas sanitarias, hilo dental, algodón, toallas húmedas, colas de cigarro y materiales plásticos). Se debe señalar que estos sólidos no deberían ser descartados por la población servida en las instalaciones sanitarias domiciliares del sistema de alcantarillado sanitario. Cuando se utiliza una secuencia de rejillas (generalmente dos), se adopta un espaciamiento entre barras gradualmente decreciente (rejillas gruesas, medianas, finas y ultra finas).

Usualmente la alternativa para la disposición final del material retenido en las rejillas (fase sólida) es la disposición en rellenos sanitarios, precedida por una etapa de remoción de humedad. Para PTARs de pequeña escala, esta disposición final puede realizarse en la propia área de la PTAR, en fosas adecuadamente preparadas y autorizadas para recibir estos desechos. Sin embargo, se debe observar que las condiciones técnicas para esta forma de disposición final son similares a las de un relleno sanitario, lo que puede implicar una excesiva demanda constructiva y operacional para una PTAR de pequeña escala. El aprovechamiento energético del material retenido en las rejillas (por ejemplo: el procesamiento conjunto en hornos de Clinker) todavía es incipiente en Brasil, pero existen reportes que indican un considerable poder calorífico inferior $(\mathrm{PCl})$ y bajo contenido de cenizas asociados a residuos retenidos en la etapa de separación con rejillas finas (BORGES, 2014). Adicionalmente, la celulosa puede constituir la mayor fracción de demanda química de oxígeno (DQQ) inerte presente en el agua residual (debido al papel higiénico) (RUIKEN et al., 2013). En algunos países desarrollados su recuperación ya ha sido realizada en escala piloto y en escala 
plena, con el objetivo del aprovechamiento energético, incorporación al asfalto, entre otros (MARCELIS \& WESSELS, 2018).

En relación a los desarenadores, dependiendo de la escala de la PTAR, se puede considerar la utilización de unidades de: i) flujo horizontal y sección rectangular o cuadrada; ii) flujo en espiral (aireado); y iii) flujo en vórtice. Las opciones de manejo del material removido (fase sólida) normalmente están asociadas al secado y posterior disposición final en rellenos sanitarios. La recuperación de arena removida para fines de preparación de concreto no estructural o reposición en lechos de secado de la PTAR todavía es incipiente, pero se puede considerar como una importante alternativa de gestión de este subproducto a corto y a mediano plazo.

Existen escasos reportes en la literatura asociados a la caracterización cualitativa de los sólidos removidos en el tratamiento preliminar, aunque se sabe que la cantidad y la composición del material recuperado en la etapa de retención de sólidos dependen principalmente del espaciamiento entre barras. Sin embargo, las características de la cuenca de contribución de la PTAR influyen en el volumen y calidad del material removido, siendo posible enumerar los siguientes factores de influencia (adaptado de WEF, 1994; WEF, 1998):

- El tamaño y el tipo de ocupación de la cuenca de contribución;

- La configuración del sistema de alcantarillado: la presencia de estaciones de bombeo disminuyen la cantidad de material retenido en las rejillas, debido a la remoción previa antes del bombeo (por ejemplo: canastas en estaciones de bombeo de agua residual y disposición del material en el fondo del pozo de succión);

- Los hábitos y la educación sanitaria de la población atendida;

- Recepción de camiones transportadores de lodo séptico, efluentes no domésticos (END), residuos de otras PTAR (como nata y lodo), residuos de estaciones de bombeo. Es importante mencionar que la recepción de esas contribuciones implica un aumento en la producción de lodo y en la formación y acumulación de nata en los reactores UASB, conforme lo presentado en la Parte 3 de esta Colección de Notas Técnicas (NTs) (LOBATO et al., 2018).

El volumen de sólidos retenidos en el cribado varía usualmente entre 6 a 50 litros por $1.000 \mathrm{~m}^{3}$ de agua residual tratada (JORDÃO \& PESSÔA, 2017). De acuerdo con una caracterización cualitativa y cuantitativa realizada por Borges (2014) en una PTAR de escala mayor (Población Equivalente $-\mathrm{PE}=258.000$ habitantes) en el interior del estado de São Paulo (Brasil), el material retenido en la etapa de cribado de material grueso (en términos de volumen y masa) fue principalmente constituido por materia orgánica putrescible (restos de alimentos, animales en descomposición, entre otros - $65 \%$ en volumen), seguido de plástico ( $17 \%$ en volumen), piedras ( $9 \%$ en volumen), tejido ( $6 \%$ en volumen) y papel ( $3 \%$ en volumen). Fue obtenida una configuración similar para la evaluación realizada en relación a los sólidos retenidos en el cribado de material fino. La composición del material retenido presentó una variación estacional significativa, indicando una fuerte influencia de la precipitación en el aumento de la cantidad de piedras. Éstas, a su vez, contenían precipitados de azufre, calcio y grasa solidificada.

En relación a la cantidad de arena presente en el agua residual cruda, esta depende de varios factores, entre ellos: i) el tipo de sistema (sistema combinado o separado de agua pluvial y residual) y el estado de las condiciones del sistema de alcantarillado y transporte de agua residual; ii) características de drenaje pluvial de la cuenca de contribución (colectores sujetos a inundación tienden a recibir mayor carga de sedimentos a través de las tapas de pozos de inspección); iii) el tipo de ocupación de la cuenca de contribución; y iv) el tipo de suelo de la cuenca de contribución y el grado de pavimentación de las calles. La cantidad de arena en sistemas separados varía usualmente entre 4 e 37 litros por $1.000 \mathrm{~m}^{3}$ de agua residual tratada (METCALF \& EDDY, 2003). 


\section{PRINCIPALES CONFIGURACIONES PARA EL TRATAMIENTO PRELIMINAR}

Las unidades que componen el tratamiento preliminar (TP) pueden ser implantadas de forma enterrada (Figura 1a), apoyada o elevada (Figura 1b) en relación al nivel del terreno. Las soluciones enterradas pueden facilitar el confinamiento del TP, tal como se muestra en la Figura 1a para el desarenador y la canaleta Parshall. Sin embargo, esta opción puede implicar una mayor dificultad operacional asociada a la rutina de limpieza de las unidades, además de que en la mayoría de las ocasiones demandan una estación de bombeo dentro de la PTAR. En esta configuración, el TP aguas arriba de la estación de bombeo puede tener un importante papel en la protección del conjunto motor-bomba, pero no hay cómo garantizar que este aspecto compense la mayor dificultad debido a la operación de las unidades enterradas. Se debe destacar que los aspectos asociados al confinamiento, extracción y tratamiento de olores en el TP son abordados en la Parte 4 de esta Colección de Notas Técnicas (BRANDT et al., 2018).

Por otro lado, la solución apoyada o elevada puede demandar mayor costo de implantación en com- paración con la solución apoyada, pero tiene la ventaja de permitir la descarga del fondo de los desarenadores por gravedad, lo que puede implicar la simplificación o eliminación de equipos electromecánicos (por ejemplo: clasificador de tornillo). Adicionalmente, el contenedor para la recolección del material retenido en el cribado puede ser colocado estratégicamente cerca del cribado, en un nivel inferior al TP. Esto contribuye para la mejora de las condiciones ergonómicas asociadas a la limpieza manual de las rejillas. Eventualmente, las unidades de cribado pueden estar conectadas a la estación de bombeo de agua residual cruda, satisfaciendo la necesidad de adoptar una estructura de control de caudales afluentes (rebalse o vertedero de excedencias) para prevenir la inundación de las rejillas. En este caso, se entiende que las rejillas son componentes de la estación de bombeo, de forma que las unidades del TP se reducirían al desarenador $y$, eventualmente, a un cribado fino y tamiz. En muchas situaciones, la elevación del TP elimina una estación de bombeo dentro de la PTAR, debido a que la ganancia en el nivel puede provenir de subir el propio nivel de la tubería de entrada, contribuyendo para que el perfil hidráulico esté a favor de la gravedad.
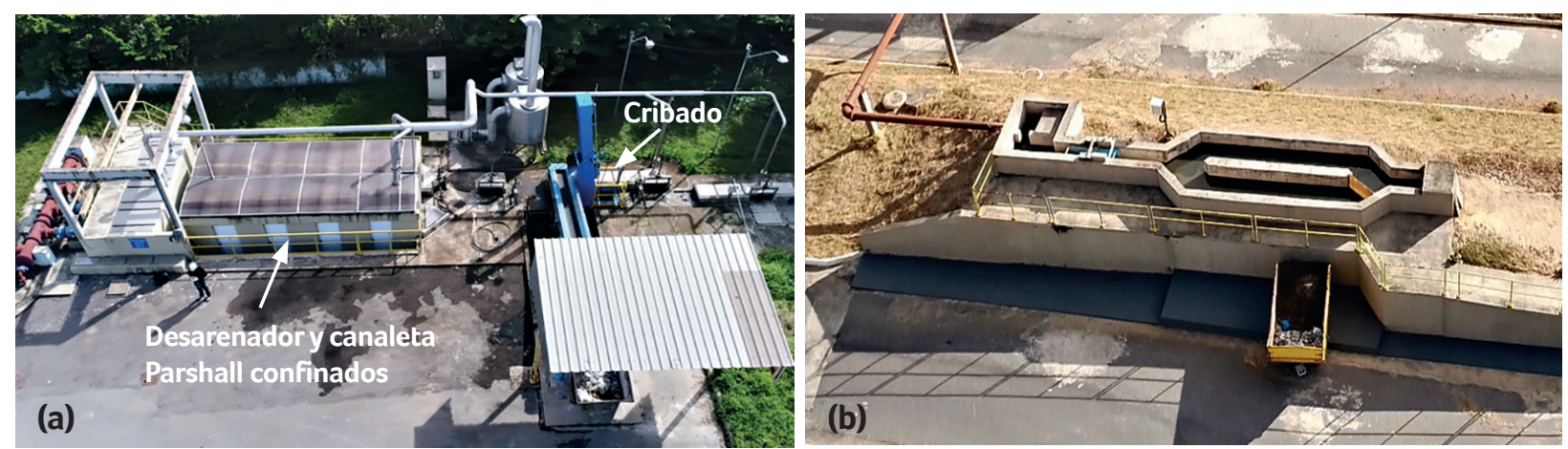

Figura 1 - Unidades del tratamiento preliminar: a) solución enterrada y confinada; b) solución elevada. 
Lastuberías (ocanales) que conducen elagua residual de las rejillas al desarenador normalmente son confeccionadas en concreto reforzado. Para PTARs de escala pequeña (Caudal - $\mathrm{Q}<20 \mathrm{~L} . \mathrm{s}^{-1}$ o PE $<10.000$ hab.), las dimensiones de estos canales pueden ser muy pequeñas, al punto de dificultar la ejecución de obra en concreto, conforme lo presentado en secciones posteriores, en el ítem "Otros aspectos importantes del diseño, construcción y operación”.

\section{DIRECTRICES PARA EL DISEÑO}

\subsection{Parámetros y criterios de dimensionamiento}

La lógica principal para el dimensionamiento de las unidades de tratamiento preliminar incluye asegurarvelocidades de flujo adecuadas para la remoción de sólidos gruesos, evitando problemas de obstrucción, desgaste y trabamiento en equipos electromecánicos, sensores y medidores de velocidad sumergidos. Sin embargo, se debe asegurar que tales velocidades no impliquen la sedimentación de materia orgánica particulada, pues esto puede propiciar la generación de olores. Las directrices de diseño para la etapa de tratamiento preliminar se encuentran detalladas en la Norma Brasileña (NBR) 12.209 (ABNT, 2011).

Los principales parámetros y criterios de dimensionamiento de las unidades que componen el tratamiento preliminar se muestran en el Cuadro 1. Las diferentes configuraciones recomendadas para el TP relacionadas a la selección de rejillas y desarenadores se presentan en la Figura 2 (en función de la población equivalente o caudal afluente a la PTAR). Se debe resaltar que estas configuraciones son originadas a partir de la experiencia operacional de PTARs en diversos estados brasileños, principalmente en las regiones sur y sureste. Estas configuraciones han sido extensamente discutidas junto a especialistas del sector de saneamiento de Brasil.
Cuadro 1 - Principales parámetros y criterios de dimensionamiento para las unidades de tratamiento preliminar

\begin{tabular}{|c|c|}
\hline \multicolumn{2}{|c|}{ Cribado (Rejillas) } \\
\hline Parámetro & Valor \\
\hline $\begin{array}{l}\text { Velocidad máxima a través de las } \\
\text { barras de rejillas para el caudal final }\end{array}$ & $1,20 \mathrm{~m} \cdot \mathrm{s}^{-1}$ \\
\hline $\begin{array}{l}\text { Inclinación de las barras en relación } \\
\text { al plano horizontal }\end{array}$ & $\begin{array}{c}45 \text { a } 60^{\circ} \text { - limpieza manual } \\
60 \text { a } 90^{\circ} \text { - limpieza mecanizada }\end{array}$ \\
\hline $\begin{array}{l}\text { Pérdida de carga mínima a ser } \\
\text { considerada (flujo aguas arriba) }\end{array}$ & $\begin{array}{c}\text { 0,15 m - limpieza manual } \\
0,10 \text { m - limpieza mecanizada }\end{array}$ \\
\hline \multicolumn{2}{|c|}{ Desarenador ${ }^{b}$} \\
\hline Parámetro & Valor \\
\hline Velocidad de flujo & 0,25 a $0,40 m \cdot s^{-1}$ \\
\hline $\begin{array}{l}\text { Profundidad mínima para } \\
\text { acumulación de material } \\
\text { sedimentado }\end{array}$ & $0,20 \mathrm{~m}$ \\
\hline Carga hidráulica superficial & 600 a $1.000 m^{3} \cdot m^{-2} \cdot d^{-1}$ \\
\hline
\end{tabular}

Nota: el caudal de dimensionamiento de las unidades del TP debe ser el caudal máximo afluente

aLos canales o tuberías aguas arriba y aguas abajo de los dispositivos de remoción de sólidos gruesos deben garantizar (por lo menos una vez al día y desde el inicio de la operación) una velocidad igual o superior a 0,40 m.s. La velocidad mínima de flujo en estos canales debe ser idealmente superior a $0,30 \mathrm{~m} \cdot \mathrm{s}^{-1}$, con el fin de evitar la disposición de arena en los canales o tuberías.

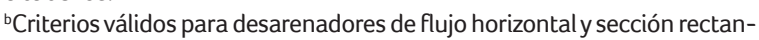
gular (tipo canal).

Fuente: adaptado de la NBR 12.209 (ABNT, 2011)

\subsection{Configuraciones recomendadas para el tratamiento preliminar}

En la NBR 12.209 no hay una recomendación explícita sobre las posibilidades de instalación de diferentes tipos de rejillas (en términos de espaciamiento entre barras y automatización de la limpieza), especialmente para PTARs de escala

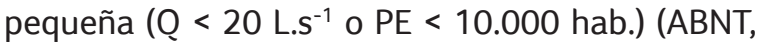
2011). Específicamente para sistemas de pequeña escala, la experiencia operacional ha sido positiva al utilizar dos rejillas consecutivas de limpieza manual, con un espaciamiento libre entre las barras de 20 y 10 mm, respectivamente (Figura 2). Es importante resaltar que la posibilidad de utilizar rejillas de limpieza manual o mecanizada para esta escala de PTAR depende del prestador de servicios, en vista del volumen de material a ser retenido o de las dificultades operacionales (NBR 12.209-ABNT, 2011). 
En el caso de PTARs cuyo caudal afluente máximo sea igual o superior a $100 \mathrm{~L} . \mathrm{s}^{-1}$, las rejillas deben ser de limpieza mecanizada.

En el caso de PTARs que utilizan reactores UASB, es imprescindible la utilización de dispositivos de remoción de sólidos gruesos con aberturas iguales o inferiores a $12 \mathrm{~mm}$ para PTARs con caudal máximo hasta de 100 L.s. $^{-1}$ (NBR 12.209 - ABNT, 2011). Las experiencias operacionales con el uso de unidades de tamizaje estático (3 a $5 \mathrm{~mm}$ de abertura de malla) en PTARs de pequeña escala, como postratamiento del cribado, indican que estas unidades son importantes para evitar obstrucciones en las líneas de alimentación de reactores UASB (en vertedores y tuberías), así como en canaletas colectoras de efluente tratado. Para estaciones cuyo caudal

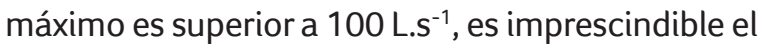
uso de tamices con aberturas iguales o inferiores a $6 \mathrm{~mm}$, según la indicación de la norma NBR 12.209 (ABNT, 2011). En este caso, las experiencias operacionales han indicado el uso preferencial de tamices con aberturas de malla de 3 a $4 \mathrm{~mm}$.

La calidad del lodo y de la nata descartadas, en términos de presencia de sólidos gruesos, también se refleja en la operación de los sistemas de deshidratación mecanizada, reduciendo la necesidad de mantenimiento debido problemas como la obstrucción de tuberías de alimentación de bombas y centrífugas, bloqueos de rotores y estatores de bombas y de mezcladores, rompimiento de sellos mecánicos, acumulación de residuos en tanques de ecualización, entre otros. Adicionalmente, una remoción más eficiente de sólidos gruesos propicia la obtención de un lodo de mejor calidad estética, lo que puede favorecer su uso agrícola, además de disminuir la formación de nata en el interior de los reactores anaerobios.

En relación a los desarenadores para sistemas de pequeña escala ( $Q$ < 20 L.s ${ }^{-1}$ o PE $<10.000$ hab.), usualmente son adoptadas unidades de flujo horizontal y sección rectangular (tipo canal - Ver Figu- ra 1b), con velocidad constante proporcionada por medio de una sección de control colocada aguas abajo del desarenador (normalmente una canaleta Parshall) y remoción no mecanizada de arena. Una opción bastante utilizada para los sistemas de pequeña y media escala ( $\mathrm{Q}<100$ L.s ${ }^{-1}$ o PE $<50.000$ hab.), principalmente en la región sur de Brasil, es el desarenador de flujo de vórtice (tipo ciclón), con remoción de arena por medio de air lift. Para sistemas de escala mayor ( $Q$ : 100 L.s $^{-1}$ o PE $\geq 50.000$ hab.) comúnmente son adoptadas las unidades de flujo horizontal y sección cuadrada en planta. En este caso la remoción de arena retenida es efectuada por medio de brazos raspadores y lavador de arena. Unidades de escala mayor requieren atención especial para asegurar una distribución uniforme de caudal afluente en toda la sección de flujo del canal, además de una cuidadosa operación para identificar la ocurrencia de flujos preferenciales y/o colmatación en puntos específicos del canal, principalmente aguas arriba de las rejillas y en el inicio de operación de las PTARs.

Específicamente en relación a los desarenadores aireados (flujo en espiral), aunque sean equipos de reconocida eficiencia para la remoción de arena, las escasas experiencias brasileñas reportadas en la literatura indican una baja eficiencia de estos equipos en relación con la remoción de grasas y aceites. Entre los posibles motivos sugeridos se puede mencionar la diferencia de viscosidad del líquido en función de la temperatura, debido a que la mayoría de equipos son diseñados para condiciones de clima temperado. Aun así, debe rescatarse que existen algunas experiencias exitosas de operación de desarenadores aireados que fueron obervados por los autores en el estado de Rio de Janeiro, Brasil. La posibilidad de remoción de grasas y aceites en la etapa de tratamiento preliminar puede ser interesante para la reducción de formación de nata en reactores UABS, que especialmente en PTARs de escala mayor representa uno de los principales problemas operacionales. 


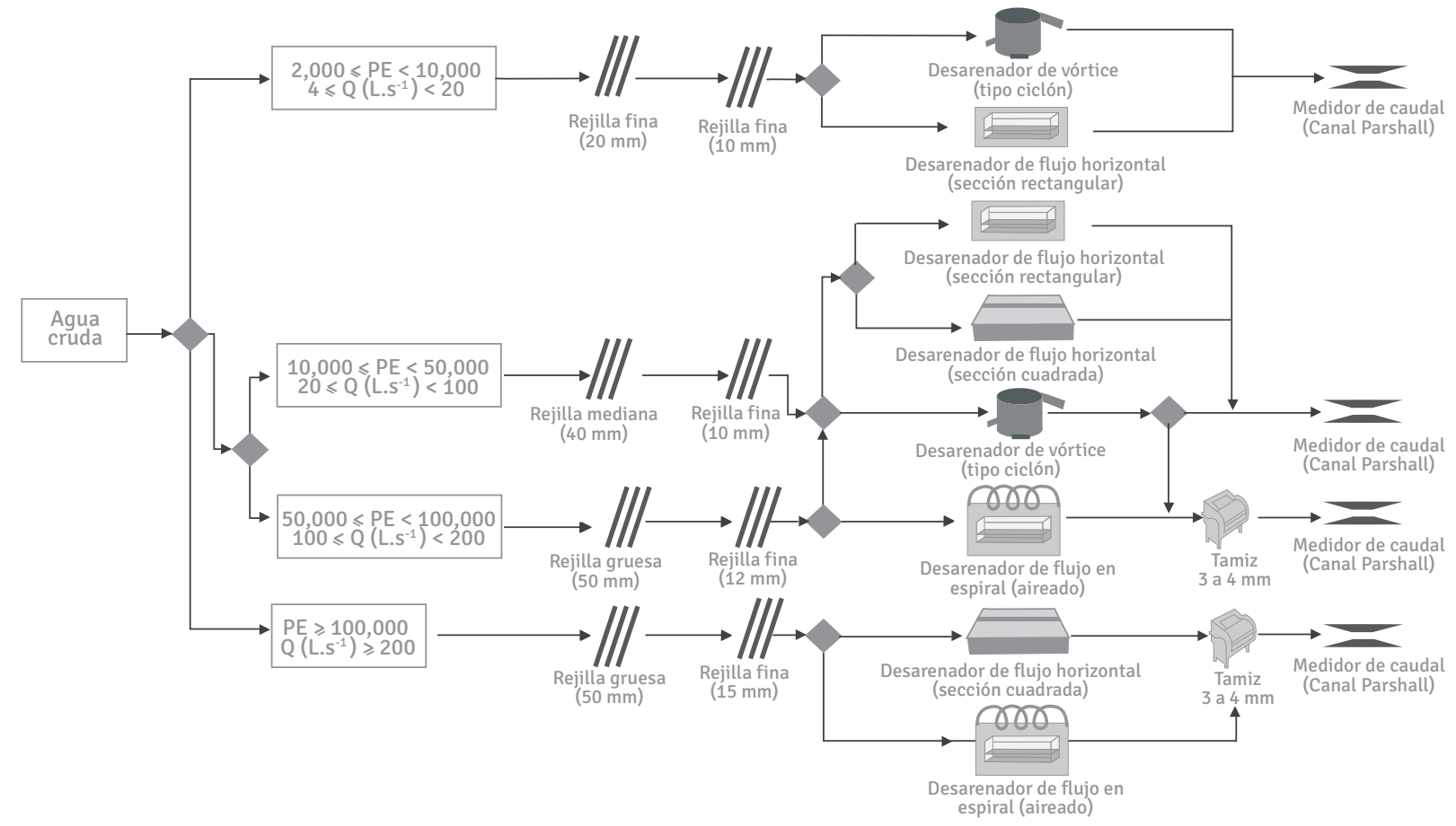

Figura 2 - Alternativas recomendadas para las unidades de tratamiento preliminar de PTARs de diferentes escalas. Nota: las equivalencias entre Q y PE fueron determinadas a partir de. El dimensionamiento de las unidades del TP debe ser realizado utilizando $Q_{\text {máx }}$

Adicionalmente a las alternativas recomendadas en la Figura 2, las posibilidades de modulación y de redundancia de las unidades del TP deben serverificadas, específicamente para PTARs de mediana y gran escala ( $Q 20$ L.s s $^{-1}$ o PE $\geq 10.000$ hab.), conforme lo indicado en el Cuadro 2. Para PTARs de pequeña escala ( $\mathrm{Q}<20$ L.s ${ }^{-1}$ o PE $<10.000$ hab.), se recomienda la colocación de un canal o tubería de desvío (by-pass) que cuente con un cribado fino (rejillas con espaciamiento entre barras igual a $20 \mathrm{~mm}$ ).

\subsection{Material retenido en las unidades del tratamiento preliminar}

Según los resultados de un estudio de 24 PTARs en la cuenca hidrográfica del Río de las Velhas (estado de Minas Gerais, Brasil), el volumen promedio de material retenido en las unidades de cribado es del orden de 4,2 litros por $1.000 \mathrm{~m}^{3}$ de agua residual tratada (Figura 3) (2,5 a 8,0 L.1.000 m ${ }^{-3}$ - percentil 25 y $75 \%$ de los datos), muy cercano al límite inferior del rango de valores reportados en la literatura, el cual está entre 6 y 50 litros por $1.000 \mathrm{~m}^{3}$ de agua residual tratada (JORDÃO \& PESSÔA, 2017). De cierta forma, el bajo volumen de material retenido en el cribado es inesperado, pues es sabido que en la mayoría de ciudades brasileñas la cantidad de sólidos presentes en el agua residual cruda es elevada. Existe la duda de si la baja retención del material en las rejillas es consecuencia de un alto espaciamiento entre las barras de las rejillas, lo que en última instancia estaría contribuyendo con la elevada presencia de sólidos en el interior de los reactores (junto con la nata y el lodo).

Para 23 de las 24 PTARs investigadas, el volumen medio del material retenido en el desarenador fue del orden de 40 litros por $1.000 \mathrm{~m}^{3}$ de agua residual tratada (Figura 3) (20 a 85 L.1.000 $\mathrm{m}^{-3}$ - percentil 25 y $75 \%$ de los datos), ligeramente superior al límite máximo de 37 litros por $1.000 \mathrm{~m}^{3}$ de agua residual tratada, reportado por Metcalf \& Eddy (2003). Esa elevada cantidad de arena puede ser atribuida a la infiltración en la red colectora y principalmente a la contribución pluvial de conexiones ilícitas, que parece ser una realidad en todo el territorio nacional brasileño. Como información complementaria, datos confiables indican que en el litoral paulista los volúmenes de arena retenidos son del orden de 60 litros por $1.000 \mathrm{~m}^{3}$ de agua residual tratada. 

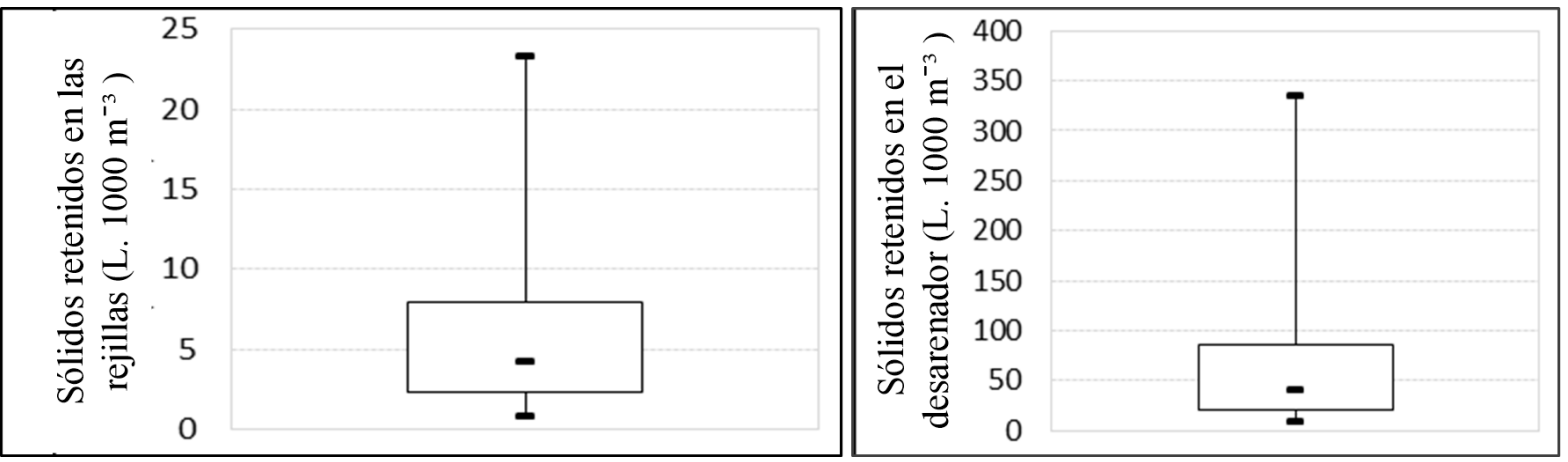

Figura 3 - Material retenido en las unidades de tratamiento preliminar de PTARs en la cuenca hidrográfica del Río de las Velhas: (a) sólidos retenidos en rejillas (L.1.000m³); y (b) sólidos retenidos en el desarenador (L.1.000m-3).

De hecho, los volúmenes de sólidos groseros y de arena retenidos en la etapa de tratamiento preliminar dependen de una serie de factores, tal como fue indicado anteriormente en estas Notas Técnicas. Estos factores deben ser cuidadosamente valorados en la etapa de diseño de las PTARs.

\section{ASPECTOS RELACIONADOS CON EL DISEÑO, LA CONSTRUCCIÓN, LA OPERACIÓN Y EL \\ MANTENIMIENTO}

Los principales aspectos a ser evaluados en el análisis de diseño de las unidades que componen el tratamiento preliminar están sintetizados en el Cuadro 2. Al enumerarlos, se buscó definir el grado de importancia asociado al cumplimiento de cada aspecto, de acuerdo con los siguientes criterios:

- Grado de importancia media - aspectos que si no son cumplidos pueden comprometer la operación y mantenimiento adecuado del sistema.

- Grado de importancia alta- aspectos cuyo cumplimiento es imprescindible para la buena operación y mantenimiento del sistema.

Cuadro 2 - Principales aspectos a evaluar en el diseño de las unidades que componen el tratamiento preliminar

\begin{tabular}{|c|c|c|}
\hline \multirow{2}{*}{ Generales } & \multicolumn{2}{|c|}{$\begin{array}{l}\text { Grado de } \\
\text { importancia }\end{array}$} \\
\hline & Alto & Medio \\
\hline $\begin{array}{l}\text { Consultar previamente a la unidad operacional y de mantenimiento electromecánico si la definición de la conceptualización del TP es adecuada } \\
\text { (enterrado, elevado, aguas arriba de la estación de bombeo, equipos, posibilidad de recibir el lodo de camiones transportadores de lodo y efluentes } \\
\text { no domésticos, forma de limpieza y vaciado de las unidades, necesidad de control de olores, entre otros). }\end{array}$ & $\mathrm{X}$ & \\
\hline $\begin{array}{l}\text { Dimensionar las unidades utilizando el caudal máximo afluente y asegurar las velocidades mínimas de flujo, de forma que se impida la } \\
\text { sedimentación indeseada de solidos orgánicos (SSV). }\end{array}$ & $x$ & \\
\hline Justificar la elección de tratamiento preliminar manual o mecanizado. & $x$ & \\
\hline $\begin{array}{l}\text { Verificar el perfil hidráulico de las unidades del TP. Deben ser consideradas las pérdidas de carga (tuberías, canales, compuertas y equipos) desde el } \\
\text { punto de control de flujo (por ejemplo: canaleta Parshall) hasta la unidad de cribado, para la definición de la lámina de agua (aguas arriba y aguas } \\
\text { abajo de cada unidad). }\end{array}$ & $X$ & \\
\hline $\begin{array}{l}\text { Prever el dispositivo de rebalse (o vertedero de excedencias) cuando el caudal afluente es superior a la capacidad de diseño de la PTAR. Nótese } \\
\text { que en PTARs en las cuales la estación de bombeo de entrada está localizada aguas abajo del TP y en PTARs donde hay riesgo de interrupción del } \\
\text { abastecimiento de energía eléctrica, deben ser colocados dos rebalses: uno antes del TP y otro conectado a un pozo de succión en la estación de } \\
\text { bombeo de entrada de la PTAR. Esta medida permite evitar la inundación de unidades colocadas aguas arriba de las estaciones de bombeo. }\end{array}$ & $X$ & \\
\hline $\begin{array}{l}\text { En la tubería de rebalse de la PTAR se deben colocar medidores de caudal que aseguren el registro de la frecuencia y la magnitud de los caudales } \\
\text { rebalsados y enviados al cuerpo receptor de agua. }\end{array}$ & & $x$ \\
\hline $\begin{array}{l}\text { Prever una válvula antirretorno (válvula check) si el rebalse es inundado, la válvula debe ser instalada cerca del tratamiento preliminary se debe } \\
\text { proveer un acceso para el mantenimiento de la válvula. }\end{array}$ & $x$ & \\
\hline $\begin{array}{l}\text { El by-pass localizado aguas arriba del tratamiento preliminar deberá contar con un dispositivo para vaciar la arena depositada en la red de } \\
\text { alcantarillado sanitario. }\end{array}$ & $x$ & \\
\hline $\begin{array}{l}\text { Para PTARs de media y gran escala ( } Q \geq 20 \text { L.s }{ }^{-1} \text { o PE } \geq 10.000 \text { hab.) se debe colocar redundancia (duplicación) de las unidades del TP (cribado, } \\
\text { desarenador y tamiz). Evaluar alternativas de modulación para la reducción de la inversión inicial. Sin embargo, se debe asegurar el mantenimiento } \\
\text { de velocidades mínimas a fin de evitar el depósito o sedimentación indeseada de sólidos. }\end{array}$ & $\mathrm{X}$ & \\
\hline
\end{tabular}




\begin{tabular}{|c|c|c|}
\hline Aspecto & $\begin{array}{r}\mathrm{Gr} \\
\mathrm{impc}\end{array}$ & $\begin{array}{l}\text { de } \\
\text { tância }\end{array}$ \\
\hline Aspectos gerais & Alto & Medio \\
\hline Para PTARs de pequeña escala (Q < $20 \mathrm{~L} . \mathrm{s}^{-1}$ o PE < 10.000 hab.) se debe instalar un canal paralelo (by-pass) con una rejilla de separación 20 mm. & X & \\
\hline $\begin{array}{l}\text { Verificar la posibilidad o viabilidad técnica y económica de utilizar cribado mecanizado y/o tamiz estático para PTARs de pequeña a media escala (Q } \\
<100 \text { L.s } \text { o }^{-1} \text { PE }<50.000 \text { hab.). }\end{array}$ & & $x$ \\
\hline $\begin{array}{l}\text { En caso de que no sea adecuado utilizar un cribado mecanizado, se debe evaluar la posibilidad de instalar rejillas manuales y secuenciales, } \\
\text { compuestas por una rejilla gruesa, media y fina. }\end{array}$ & & $\mathrm{x}$ \\
\hline $\begin{array}{l}\text { Prever el sistema de recolección, almacenamiento, transporte y disposición final de los residuos sólidos retenidos en el TP, considerando la rutina } \\
\text { operacional (frecuencia de remoción y destino final) e incomodidad de la población cercana a la PTAR debido a la generación de olores. }\end{array}$ & $\mathrm{X}$ & \\
\hline $\begin{array}{l}\text { Prever compuertas aguas arriba y aguas abajo de las unidades, de forma que permitan la entrada, aislamiento, limpieza y desvío (by-pass) de las } \\
\text { aguas residuales. }\end{array}$ & $x$ & \\
\hline En la especificación de compuertas y equipos, se debe considerar el uso de materiales resistentes a la corrosión. & $\mathrm{x}$ & \\
\hline $\begin{array}{l}\text { Para las estaciones de bombeo, se debe revisar la profundidad de pozo de succión, siendo preferiblemente menor a 8,0 m. En caso de pozos } \\
\text { superiores a 8,0 m deberán ser previstas condiciones de acceso para el mantenimiento y limpieza (plataformas intermedias, rampa, escalera, } \\
\text { barandas perimetrales, entre otros). Tanto para profundidades inferiores a 8,0 m como para superiores a 8,0 m se deben asegurar condiciones } \\
\text { adecuadas para el acceso y limpieza. }\end{array}$ & $x$ & \\
\hline $\begin{array}{l}\text { Prever contenedores menores en PTARs de pequeña escala, con el fin de no acumular residuos por largos períodos de tiempo con la consecuente } \\
\text { generación de olores. }\end{array}$ & & $\mathrm{x}$ \\
\hline $\begin{array}{l}\text { Evaluar la necesidad de prever dispositivos para cubrir los contenedores de residuos en función de las condiciones pluviométricas locales y de la } \\
\text { necesidad de control de olores. }\end{array}$ & $x$ & \\
\hline Considerar la colocación de asfalto o aceras en el perímetro de las unidades durante el diseño paisajístico y urbanístico del tratamiento preliminar. & $\mathrm{X}$ & \\
\hline $\begin{array}{l}\text { Garantizar el acceso adecuado para vehículos utilizados durante la operación y retiro de contenedores, incluyendo los siguientes aspectos: } \\
\text { inclinación adecuada de las rampas de acceso; área de maniobra suficiente y radios de curvas adecuados para el tránsito de camiones; base y } \\
\text { pavimento adecuado para el tráfico de vehículos previsto. }\end{array}$ & $x$ & \\
\hline $\begin{array}{l}\text { Prever el acceso adecuado de los operadores para que realicen las actividades de rutina y también de mantenimiento electromecánico de las } \\
\text { unidades (labores tales como lubricación, retiro de motores, tornillos, entre otros). }\end{array}$ & $\mathrm{x}$ & \\
\hline Considerar la recolección y el envío de los lixiviados de los contenedores hacia el afluente de la PTAR. & $\mathrm{X}$ & \\
\hline $\begin{array}{l}\text { En PTARs que reciban efluentes de camiones transportadores de lodo, nata, lodo de otras PTARs y de estaciones de bombeo, se debe considerar } \\
\text { la instalación de una unidad específica para su recepción y control (por ejemplo: tanque de homogenización), considerando el impacto en la } \\
\text { producción de lodo en el reactor UASB (ver Nota Técnica } 3 \text { - Lobato et al., 2018). }\end{array}$ & $x$ & \\
\hline $\begin{array}{l}\text { Garantizar la compatibilidad entre los equipos previstos en el diseño y aquellos que efectivamente son adquiridos, con el fin de evitar adecuaciones } \\
\text { en la estructura civil y electromecánica de las unidades del tratamiento preliminar durante las fases de construcción y operación. }\end{array}$ & $\mathrm{x}$ & \\
\hline Cribado & Alto & Medio \\
\hline $\begin{array}{l}\text { Para la estimación de sólidos gruesos y arena retenida se deben utilizar datos operacionales representativos de la realidad local (verificar las } \\
\text { características de la cuenca de contribución, como la ocupación y la actividad económica). }\end{array}$ & $\mathrm{x}$ & \\
\hline $\begin{array}{l}\text { En el caso de las unidades mecanizadas, se debe prever un canal paralelo con una rejilla de limpieza manual con el mismo espaciamiento entre } \\
\text { barras que la unidad mecanizada, de forma a contar con un respaldo de la unidad mecanizada. }\end{array}$ & $\mathrm{x}$ & \\
\hline $\begin{array}{l}\text { El cribado deberá ser realizado con barras de sección rectangular. No se deben utilizar barras de sección circular o barras horizontales (para el } \\
\text { soporte de las barras principales), pues dificultan el libre paso del rastrillo utilizado para la limpieza. }\end{array}$ & $\mathrm{x}$ & \\
\hline $\begin{array}{l}\text { Para el tratamiento preliminar instalado aguas abajo de una estación de bombeo, se deberá prever cribado grueso aguas arriba de la estación } \\
\text { de bombeo (notar que el espaciamiento entre barras debe ser inferior al diámetro del rotor de la bomba, evitando el paso de sólidos gruesoss de } \\
\text { dimensiones mayores). Adicionalmente, se deberá evaluar la posibilidad de implantar cribado medio y fino aguas arriba de la estación de bombeo. }\end{array}$ & $x$ & \\
\hline $\begin{array}{l}\text { Para PTARs con deshidratación mecanizada de lodo y/o de nata, se debe prever una etapa de tamizaje con malla de hasta } 6 \mathrm{~mm} \text { (preferiblemente } \\
3 \text { o } 4 \mathrm{~mm} \text { ) en el tratamiento preliminar, asegurando una adecuada operación y minimización de paros de esas unidades (por ejemplo: agitador de } \\
\text { ecualización, bombas de alimentación de equipos de deshidratación). }\end{array}$ & $x$ & \\
\hline $\begin{array}{l}\text { En PTARs de mayor escala que posean cribado mecanizado, se debe evaluar la posibilidad de utilizar dispositivos de compactación para los residuos } \\
\text { removidos, con el objetivo de reducir el volumen y la liberación de lixiviados en los contenedores, reducir olores y mejorar las características del } \\
\text { material para su disposición final. }\end{array}$ & & $x$ \\
\hline Desarenador & Alto & Medio \\
\hline $\begin{array}{l}\text { Cuando el ancho del desarenador sea inferior a } 30 \mathrm{~cm} \text {, se debe evaluar y proponer alternativas para su construcción, por ejemplo: un desarenador } \\
\text { del tipo flujo en vórtice o confección de equipos de limpieza a la medida, compatibles con las dimensiones del canal También se recomienda } \\
\text { adoptar soluciones prefabricadas en materiales alternativos (por ejemplo: fibra de vidrio y polipropileno - PP) }\end{array}$ & & $x$ \\
\hline $\begin{array}{l}\text { Para desarenadores de limpieza manual, se debe verificar la existencia de un desnivel adecuado para la acumulación de arena en función de la } \\
\text { frecuencia de limpieza adoptada por la operación. }\end{array}$ & $\mathrm{x}$ & \\
\hline Donde la topografía lo permita, se debe utilizar el descarte de arena en el fondo, de manera que se reduzca el gasto en camiones cisterna. & & $\mathrm{x}$ \\
\hline $\begin{array}{l}\text { Para los desarenadores mecanizados de sección cuadrada, el moldeo de la forma externa del raspador debe ser realizado dentro del propio } \\
\text { desarenador en el que estará inscrito (contemplando redondeos o chaflanes en los vértices), utilizando el propio brazo del raspador. }\end{array}$ & $\mathrm{x}$ & \\
\hline $\begin{array}{l}\text { Para los desarenadores de sección cuadrada, se debe garantizar una distribución uniforme de caudal en el ancho de la unidad. Además de tener un } \\
\text { dimensionamiento hidráulico adecuado en el canal de acceso, deberá existir la posibilidad de regular el caudal en cada punto de entrada. }\end{array}$ & $x$ & \\
\hline $\begin{array}{l}\text { Para los desarenadores de sección cuadrada, se debe garantizar la posibilidad de acceso para la limpieza del canal afluente, considerando } \\
\text { que en algunas situaciones puede haber sedimentación de arena en los extremos de ese canal debido a velocidades inferiores a las } \\
\text { mínimas previstas en el diseño. }\end{array}$ & $\mathrm{x}$ & \\
\hline $\begin{array}{l}\text { Para los desarenadores de sección cuadrada, evaluar la posibilidad de prever descarga de fondo para el vaciado de las unidades, con el fin } \\
\text { de permitir la limpieza y el mantenimiento del brazo raspador y del dispositivo clasificador. }\end{array}$ & $\mathrm{X}$ & \\
\hline
\end{tabular}


Nótese que la mayoría de los aspectos anteriormente listados fueron categorizados como de importancia alta. Este hecho refleja la importancia del tratamiento preliminar para el adecuado acondicionamiento del agua residual antes de su ingreso a los reactores UASB. En síntesis, un adecuado tratamiento preliminar influenciará positivamente en: i) el mantenimiento de las estructuras de distribución de caudal y canaletas colectoras del efluente, evitando obstrucciones; ii) la reducción de sedimentación/acumulación de arena en el fondo de los reactores UASB; iii) la gestión del lodo, debido a que será posible obtener un lodo con menor contenido de residuos sólidos y arena, y de mejor calidad estética; iv) la gestión de nata, sobre todo debido al hecho de que, cuando no son removidos adecuadamente en el tratamiento preliminar, algunos materiales tienden a flotar en el interior del reactor y a acumularse en la parte superior del separador trifásico; y iv) la reducción significativa de problemas habituales en la operación de unidades de procesamiento mecanizado de lodo y nata, como el paro por obstrucciones de tuberías, falla o pérdida de rendimiento de equipos por desgaste.

\subsection{Emisiones de olores en el tratamiento preliminar}

Cuando los canales del TP no estén confinados, el perfil hidráulico de diseño y la implantación de las unidades debe evitar la formación de turbulencias, minimizando el desprendimiento de emisiones odorantes y con potencial de corrosión. Cuando los canales del TP estén confinados, se debe considerar una llegada de agua residual en régimen turbulento (por ejemplo: uso de tuberías con descarga encima del nivel de agua máximo operacional), de modo a facilitar el desprendimiento, emisión y tratamiento de los gases disueltos en el agua residual afluente. Además, deben ser utilizados materiales resistentes a la corrosión y se deben proteger las estructuras de concreto en sitios de conocida emisión de gases, tal como se indica en la Parte 4 de esta Colección de NTs (BRANDT et al., 2018).

La emisión de olores en el tratamiento preliminar en PTARs de pequeña escala también está asociada a la acumulación del material retenido en las rejillas cuando se descarga en los contenedores. Se recomienda el uso de contenedores de menor volumen, para que el material depositado no se mantenga almacenado por mucho tiempo. Eventualmente, la aplicación de cal virgen para controlar la emisión de olores puede ser necesaria (Figura 4 a). En estos casos, es necesario prever un sitio adecuado para el almacenamiento de esos productos en la PTAR. Por otro lado, para PTARs de gran escala ( $Q \geq 100$ L.s ${ }^{-1}$ o PE $\geq 50.000$ hab.), pueden ser requeridas medidas de cobertura para los contenedores a base de materiales resistentes (por ejemplo: tapas con bisagra y fijación de lonas) y el cumplimiento de aspectos ergonómicos (por ejemplo: revisión del peso). El acceso de camiones para el retiro de contenedores también debe ser considerado en la etapa de diseño y construcción de la PTAR (por ejemplo considerar: declividad de rampas, radios de curvas, capacidad de soporte de pavimento, área de maniobra).

Otro aspecto importante es garantizar las velocidades mínimas de flujo en los canales aguas arriba y aguas abajo del cribado y del desarenador. Se recomienda que sean superiores a 0,30 $\mathrm{m} . \mathrm{s}^{-1}$ para evitar el depósito indeseable de sólidos en esas estructuras, según se ejemplifica en la Figura 4b. Se debe prestar especial atención a los posibles cambios de sección transversal en el transcurso de estos canales. La eventual acumulación de materiales en esos puntos implica la emisión de malos olores, asociados a la descomposición de los sólidos orgánicos indebidamente retenidos, además de que puede comprometerse el funcionamiento de rejillas y compuertas. 

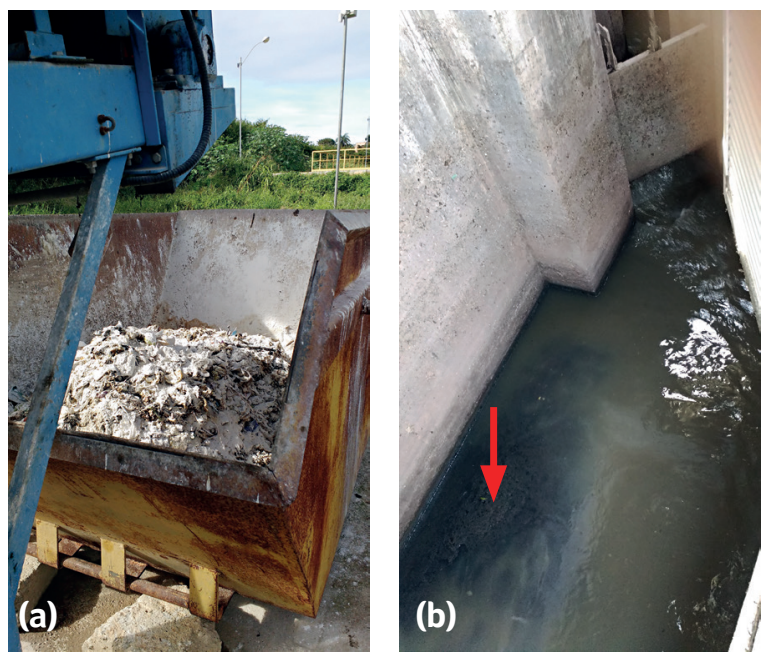

Figura 4 - (a) Manejo del material removido en la etapa de cribado, con aplicación de cal; (b) acumulación de sólidos en el canal del afluente al desarenador.

\subsection{Bombeo y distribución de caudal}

La etapa del bombeo del agua residual que ingresa a la PTAR hacia el reactor UASB es un punto crucial para el adecuado desempeño de los reactores anaerobios. En los casos en los que hay necesidad de bombear el agua residual afluente hacia los reactores UASB, los diseños normalmente contemplan sólo dos bombas, siendo una para relevo y reserva; especialmente en plantas de pequeña y mediana escala (Q < 100 L.s $^{-1} \mathrm{o}$ $\mathrm{PE}<50.000$ hab.). Cuando los reactores son expuestos a condiciones de sobrecarga hidráulica debido al bombeo constante de un caudal igual o superior al caudal máximo para el cual fueron diseñados, es posible que se presente pérdida de eficiencia de la retención de sólidos y remoción de materia orgánica.

Tal como se establece en el ítem 6.4.2 de la norma NBR 12.209, en el caso de alimentación por estación de bombeo, el caudal máximo de bombeo no puede exceder más del $25 \%$ del caudal máximo de agua residual afluente (ABNT, 2011). En este caso, se recomienda el uso de bombas con variadores de velocidad (variadores de frecuencia) o la instalación de un mínimo de tres bombas, siendo una para relevo y reserva. Adicionalmente, se re- comienda la instalación de un medidor de caudal después de la estación de bombeo, con la intención de caracterizar el hidrograma del caudal afluente en el reactor UASB. Esta medida es de gran importancia, sobre todo cuando se remplazan los equipos de bombeo, actividad de mantenimiento en la que es común instalar bombas con puntos de operación diferentes.

Respecto a las cajas de distribución de agua residual afluente a los reactores UASB, es común observar estructuras con el fondo plano o con poca inclinación (Figura 5a), lo que propicia la acumulación de arena y/o sólidos, especialmente cuando ocurren fallas en el tratamiento preliminar. En este sentido, se recomienda el uso de cajas con fondo inclinado (Figura 5b). También se recomiendan estructuras confeccionadas en materiales que permitan un mejor acabado (por ejemplo: fibra de vidrio - Figuras $5 a$ y $5 b$ ), así como el correcto dimensionamiento y verificación de las láminas de agua en los vertedores triangulares para los caudales máximos de bombeo. Estos son aspectos importantes para garantizar una distribución uniforme de caudal dentro de los compartimientos que alimentan los tubos de distribución. Además, los tubos conectados al fondo de las cajas de distribución deben poseer diámetro de $75 \mathrm{~mm}$, ya que mientras garantizan una velocidad descendente adecuada, también son suficientemente grandes para evitar obstrucciones frecuentes (obviamente cuando se tiene un funcionamiento satisfactorio del TP). Es importante garantizar la correcta fijación de los tubos a las estructuras de distribución, pues éstos pueden soltarse debajo de la losa sin que el operador perciba que un tubo está desconectado. En ese caso, el agua residual afluente entraría en el compartimiento de sedimentación del reactor UASB, en vez de ser distribuida en el fondo de la unidad. 

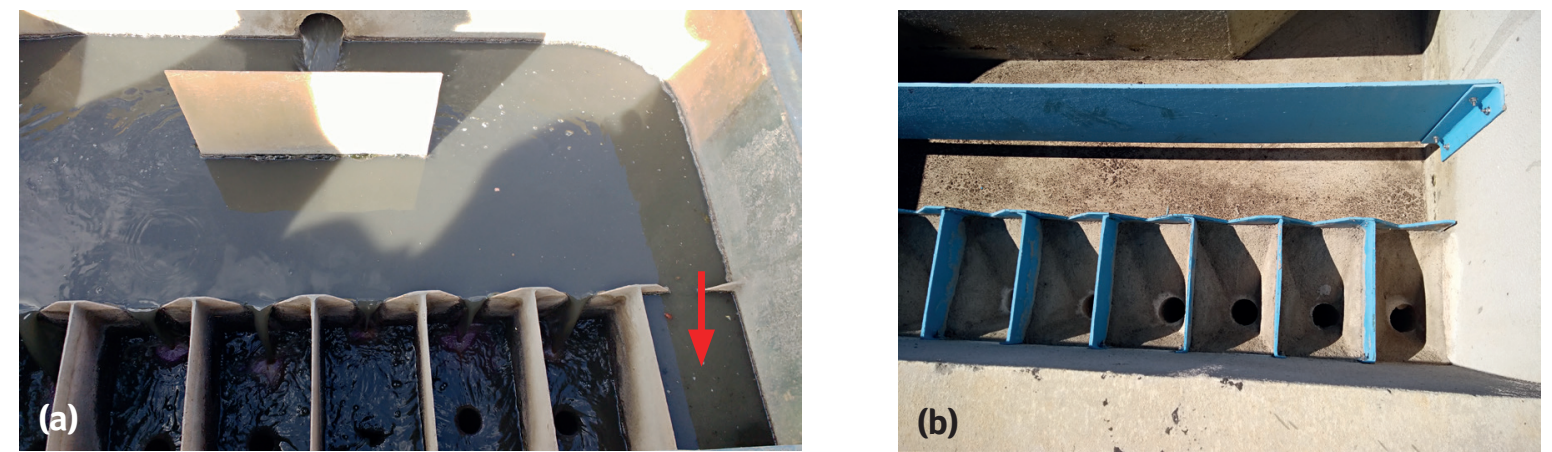

Figura 5 - Detalles de compartimientos de distribución de caudal afluente a los reactores UASB: a) Caja con fondo plano, resaltando la colmatación de un tubo; b) caja con fondo inclinado.

\subsection{Otros aspectos importantes del diseño, la construcción y la operación}

Específicamente para PTARs de pequeña escala ( \& $<20$ L.s s $^{-1}$ o PE $<10.000$ hab.), las dimensiones de algunas unidades del TP son diminutas. En el caso de los canales afluentes y efluentes al cribado y al desarenador, resulta difícil cumplir con las condiciones ideales de flujo previstas en la norma NBR 12.209 (ABNT, 2011). Por otro lado, el uso de estructuras de concreto armado colado en sitio puede no ser una solución adecuada para la construcción de las unidades, debido a la complejidad asociada al retiro de las formaletas y a la dificultad para cumplir con el recubrimiento mínimo de las armaduras ( $5 \mathrm{~cm}$ - NBR 6.118 - ABNT, 2014). En este caso, soluciones con estructuras prefabricadas en materiales alternativos pueden ser una opción adecuada, por ejemplo: estructuras de plástico reforzado con fibra de vidrio o de polipropileno.

La preocupación con criterios ergonómicos, de salud y de seguridad ocupacional debe ser integrada al diseño y la construcción de las unidades del TP, considerando por ejemplo: la instalación de barandas, escaleras de acceso resistentes a la corrosión, tomas de agua potable, aberturas mínimas para el paso de operadores y herramientas adecuadas para las operaciones de limpieza y mantenimiento. La previsión de dispositivos de vaciado de las unidades también permite que las actividades de limpieza sean más ágiles y simples.

Es de fundamental importancia que el diseño considere las especificaciones de los equipos que serán efectivamente adquiridos e implantados, tales como: pérdida de carga efectiva, necesidad de disminución del nivel de agua, demanda de agua para limpieza, descarga de drenajes, apoyos para tornillos clasificadores y rejillas, accesos para mantenimiento electromecánico y limpiezas, entre otros. La compatibilidad entre los equipos previstos en el diseño y aquellos que efectivamente serán adquiridos es crucial para evitar cambios en la estructura civil y electromecánica de las unidades del TP durante las fases de construcción y operación. Estas adecuaciones durante la construcción y operación pueden representar costos significativos.

Finalmente, es importante prestar atención a problemas constructivos recurrentes, tanto de construcción como de instalación de equipos, tales como: equipos instalados con desniveles (compuertas, brazos raspadores de desarenadores, clasificadores de arena) y equipos que reducen la sección transversal del canal con elementos laterales. 


\section{AGRADECIMIENTOS}

Los autores agradecen el apoyo recibido del Consejo Nacional de Desarrollo Científico y Tecnológico - CNPq (Conselho Nacional de Desenvolvimento Científico e Tecnológico), de la Coordinación de Mejoramiento de Personal de Nivel Superior - CAPES (Coordenação de Aperfeiçoamento de Pessoal de Nivel Superior), de la Fundación de Amparo a la Investigación del Estado de Minas Gerais - FAPEMIG (Fundação de Amparo à Pesquisa do Estado de Minas Gerais) y del Instituto Nacional de Ciencia y Tecnología en Plantas Sostenibles de Tratamiento de Aguas Residuales Urbanas (INCT ETEs Sustentáveis por sus siglas en portugués).

Este trabajo forma parte de la serie de publicaciones del Instituto Nacional de Ciencia y Tecnología en Plantas Sostenibles de Tratamiento de Aguas Residuales Urbanas (INCT ETEs Sustentáveis por sus siglas en portugués).

\section{REFERENCIAS BIBLIOGRÁFICAS}

ABNT - Asociación Brasileña de Normas Técnicas. NBR 12209: Elaboração de projetos hidráulico-sanitários de estações de tratamento de esgotos. $2^{a}$ edición. Rio de Janeiro, 2011. 57 p.

ABNT - Asociación Brasileña de Normas Técnicas. NBR 6118: Projeto de estruturas de concreto - Procedimento. $3^{\text {a }}$ edición. Rio de Janeiro, 2014. $238 \mathrm{p}$.

BORGES, N. B. Aproveitamento dos resíduos gerados no tratamento preliminar de estações de tratamento de esgoto. 2014. 238 p. Tesis (Doctorado en Ciencias: Ingeniería Hidráulica y Saneamiento). Universidad de São Paulo, São Paulo, 2014.
BRANDT, E. M. F., SANTOS, J. M. B., SOUZA, C. L., POSSETTI, G. R. C., BRESSANI-RIBEIRO, T., CARVALHO-JÚNIOR, A. N., CHERNICHARO, C. A. L. Contribución para el perfeccionamiento del diseño, la construcción y la operación de reactores UASB aplicados al tratamiento de aguas residuales urbanas - Parte 4: Control de corrosión y emisiones odorantes. Revista DAE - edición especial, v. 66, n. 214, p. 56-72, 2018.

JORDÃO, E.P., PESSÔA, C.A. Tratamento de esgotos domésticos. $8^{\text {a }}$ edición. Rio de Janeiro, ABES, 2017.

MARCELIS, P., WESSELS, C. Recovery and valorisation of cellulose from sewage: the route to circularity. In: 12th European Waste Water Management Conference, 2018, Manchester, UK. Anais. Manchester, UK, 2018.

METCALF \& EDDY, Wastewater Engineering: Treatment and Reuse. 4th edición. New York, NY: McGraw-Hill, 2003. 1819 p.

LOBATO, L. C. S., BRESSANI-RIBEIRO, T., SILVA, B. S., FLÓREZ, C. A. D., NEVES, P. N. P., CHERNICHARO, C. A. L. Contribución para el perfeccionamiento de diseño, la construcción y la operación de reactores UASB aplicados al tratamiento de aguas residuales urbanas - Parte 3: Gestión de lodo y nata. Revista DAE - edición especial, v. 66, n. 214, p. 30-55, 2018.

RUIKEN, C.J., BREUER, G., KLAVERSMA, E., SANTIAGO, T., VAN LOOSDRECHT, M. Sieving wastewater - Cellulose recovery, economic and energy evaluation. Water Research, v. 47, p. 43-48, 2013.

WEF - Water Environment Federation. Preliminary Treatment for Wastewater Facilities. Manual of Practice OM-2. Alexandria, VA. 1994.

WEF - Water Environment Federation. Design of Municipal Wastewater Treatment Plants. Manual of Practice n. 8, ASCE Manual and Report on Engineering Practice n. 76. Alexandria, VA. 1998. 